\title{
AVALIAÇÃO DA QUALIDADE DA ÁGUA DE CHUVA DO FENÔMENO FIRST FLUSH E DE VOLUMES ARMAZENADOS EM RESERVATÓRIOS DE SISTEMAS DE ÁGUAS PLUVIAIS NA CIDADE DO RIO DE JANEIRO-RJ
}

\author{
ASSESSMENT OF RAINWATER QUALITY OF FIRST FLUSH AND STORED VOLUMES IN \\ RESERVOIRS TO ANALYSIS OF POLLUTANT CONCENTRATION CURVES FROM
}

RAINWATER SYSTEMS IN THE CITY OF RIO DE JANEIRO-RJ

Letícia Delduque Alves ${ }^{a}$, Caroline Moreira de Souza ${ }^{a}$, Jaqueline Costa Areas de Almeida ${ }^{a}$, Giovana Proença Bastos $^{a}$, Ryan Rodrigues Domingos ${ }^{a}$, Gabrielle Nunes da Silva ${ }^{a}$, Daniele Maia Bilaa,

Alfredo Akira Ohnuma Jra

aUniversidade do Estado do Rio de Janeiro

\begin{abstract}
leticiadelduque@gmail.com,moreiracaroline98@gmail.com,jaqueline.areas@gmail.com,giovana proenca@hotmail.com. ryan.domingos10@gmail.com, gabii.nunes2014@gmail.com,

danielebilaueri@eng.ueri.br,akira@eng.ueri.br
\end{abstract}

Submissão: 23 de janeiro de 2021 Aceitação: 1 de julho de 2021

\section{Resumo}

Este trabalho tem como objetivo principal analisar a qualidade da água da chuva a partir da concentração de poluentes depositados em amostras de volumes iniciais precipitados e de volumes armazenados em dois sistemas de águas pluviais, de características distintas, situados na cidade do Rio de Janeiro-RJ, Brasil. A metodologia consistiu em analisar os parâmetros: turbidez, sólidos totais dissolvidos (STD), cloreto e alcalinidade em amostras mensais coletadas nos anos de 2017, 2018 e 2019, e em apresentar as curvas de massa de poluentes em função das vazões afluentes e do cálculo da concentração média desses poluentes dos eventos analisados. As curvas elaboradas para os parâmetros turbidez e cloreto mostram a carga total de poluentes e, também, a variação da qualidade da água de chuva em decorrência da lavagem produzida pelos volumes iniciais precipitados. Os valores médios obtidos em relação à turbidez foram: no CAP1: 34,97 UNT no ponto (FF) e 0,65 UNT no ponto (RR); no CAP2: 20,43 UNT no (FF) e 0,92 UNT no (RR). A partir desses resultados, o trabalho conclui que a intensidade da chuva e o tipo do material de captação das águas pluviais influenciam na remoção de poluentes depositados na superfície do telhado e que a qualidade de amostras coletadas em sistemas de águas pluviais melhora significativamente com a utilização de filtros de retenção de sedimentos e do descarte dos volumes iniciais precipitados.

Palavras-chave: Poluentes; Qualidade da água de chuva; Águas pluviais.

\section{Abstract}

The main objective of this work was to analyze the quality of rainwater from pollutant concentration curves deposited in samples of initial precipitated volumes and stored in two rainwater systems, with different characteristics, located in the city of Rio de Janeiro-RJ, Brazil. Monthly samples collected in the years 2017, 2018 and 2019 of the parameters turbidity, total dissolved solids (TDS), chloride and alkalinity were analyzed, with the results of the pollutant mass curves as a function of affluent flows and the concentration calculation average pollutant at the event. The results indicate the evidence of the total load of pollutants and the behavior of the quality of rainwater due to the variation in the washing of the initial volumes precipitated in the turbidity and chloride curves. The intensity of the rain and the type of rainwater catchment material influence the variability in the removal of pollutants deposited on the roof surface and the quality of water samples collected in rainwater systems improves significantly with the use of sediment retention filters and disposal of 
precipitated initial volumes, according to the average values obtained by the turbidity of 34.97 UNT at the point (FF) to 0.65 UNT at the point (RR) and from $20.43 \mathrm{UNT}$ at (FF) to $0.92 \mathrm{UNT}$ at (RR), at CAP1 and CAP2 systems, respectively.

Keywords: Pollutants; Rainwater Quality; Rainwater.

\section{INTRODUÇÃO}

Alterações no ciclo hidrológico são resultados de processos de urbanização e podem ter consequências prejudiciais para a população, como o aumento dos volumes do escoamento superficial e impactos na poluição difusa ocasionado a ampliação de áreas impermeáveis. (VASCONCELOS; MIGUEZ; VAZQUEZ, 2016) Nas últimas décadas, eventos pluviométricos extremos têm se tornado frequentes, em resposta às alterações do clima e aos processos de uso e ocupação do solo. (SILVA; CRUZ; AMARAL, 2016) Uma das medidas de avaliação dos impactos de natureza hidrológica em regiões metropolitanas é o monitoramento de sistemas de águas pluviais relacionados aos sistemas de drenagem urbana.

No Brasil, o aproveitamento da água de chuva para fins não potáveis tem sido cada vez mais utilizado como alternativa de abastecimento de água, sobretudo em regiões de estiagens prolongadas. A NBR no 15.527/2019 (ABNT, 2019) estabelece as condições mínimas para o uso da água da chuva quanto aos parâmetros de sua qualidade para fins não potáveis. A vida útil dos sistemas de aproveitamento de água de chuva em edificações é estabelecida para prevenir danos à saúde dos usuários e para orientar projetos de sistemas para essa finalidade. (NAKADA; MORUZZI, 2014)

A partir da análise dos parâmetros físicoquímicos pode-se avaliar a qualidade das águas pluviais para usos domésticos, como por exemplo, a irrigação de jardins. (GIKAS; TSIHRINTZIS, 2012) A qualidade do volume armazenado depende de fatores como: o tipo de materiais e telhados usados para a captação e armazenamento da água da chuva, características físicas e dinâmicas da área de estudo e da concentração de poluentes carregados durante um evento de precipitação ou pelo escoamento superficial no telhado. Esses poluentes são de origem antrópica, como a circulação de carros, e natural, como a deposição atmosférica. A relação da carga de poluentes atmosféricos acumulados em dias secos anteriores ao evento analisado sugere que períodos de estiagem prolongados tendem a possuir uma carga maior de poluentes no próximo evento de chuva. (GUNAWARDANA et al., 2012)

Para o estudo dos parâmetros de qualidade de águas pluviais, em diferentes espaços e tempos, recomenda-se avaliar o regime de chuvas do local e a amostragem de sua vazão, tendo em vista a insuficiência de dados de concentração de poluentes. (PINTO, 2016) Muitas vezes, as águas pluviais são consideradas como não poluídas, porém, em regiões densamente urbanizadas e industriais, podem apresentar $\mathrm{pH}$ baixo $\mathrm{e}$ concentrações significativas de poluentes. Portanto, sua qualidade é influenciada por diversos fatores, os decorrentes da urbanização, como as condições ambientais do local, e os meteorológicos. Outros fatores também influenciam: a coleta, o armazenamento e o tipo da superfície do telhado de captação. A qualidade da água da chuva é afetada durante a lavagem do telhado e pelo carreamento de partículas dissolvidas e em suspensão na atmosfera. (GIMENES, 2017)

A poluição de origem difusa, proveniente da lavagem e transporte de poluentes da atmosfera, de sua deposição na superfície e do escoamento superficial tende a ter uma maior concentração no início dos eventos pluviométricos. Sendo assim, a intensidade e a duração de um evento têm grande influência nas cargas de poluentes existentes. (GOMES, 2014) A carga da poluição durante uma lavagem atmosférica é representada pela concentração média do evento (CME). Esse índice proporciona uma estimativa do volume total de cada poluente. (OUYANG et al., 2015; HAUPT, 2009) O modelo da CME pode ser aplicado em qualquer parâmetro de qualidade de um evento chuvoso, sendo adequado para o estudo da água de chuva que sofre o escoamento superficial em áreas urbanas. (DIDONET, 2017)

Este trabalho teve como objetivo principal analisar a qualidade da água da chuva a partir da concentração de poluentes depositados em amostras de volumes armazenados no dispositivo de separação inicial, denominado first flush, e no reservatório principal, acumulados em diferentes sistemas de águas pluviais, situados na cidade do 
Rio de Janeiro-RJ, Brasil.

\section{METODOLOGIA}

\section{Área de estudo e descrição dos sistemas de águas pluviais}

A análise da qualidade da água da chuva foi realizada a partir da coleta de volumes armazenados em dois sistemas de captação e armazenamento de águas pluviais (CAP), intitulados CAP1 e CAP2, localizados na região centro-norte da cidade do Rio de Janeiro-RJ. Com um padrão de ocupação de áreas densamente urbanizadas, de comércio e residências, a região apresenta um tráfego intenso de veículos e tem proximidade com o maciço da Floresta da Tijuca (Figura 1). A localização dos sistemas monitorados de águas pluviais ocorre em função da morfologia da área urbana densamente ocupada, da tipologia da superfície do telhado de captação e da capacidade de armazenamento de coletores de água de chuva.

O sistema de captação e armazenamento de águas pluviais CAP1 (Figura 2a) está localizado no Rio Comprido, na região central da cidade, nas dependências do Instituto de Aplicação Fernando Rodrigues da Silveira, da Universidade do Estado do Rio de Janeiro (CAp-UERJ), próximo ao elevado Engenheiro Freyssinet, uma área com intensa liberação de poluentes atmosféricos oriundos de cerca de 20.600 veículos, em média, por dia útil no local (PREFEITURA DO ESTADO DO RIO DE JANEIRO, 2017). O bairro Rio Comprido apresenta $62 \%$ de sua área total urbanizada, segundo a Prefeitura da Cidade do Rio de Janeiro (2018). Com uma área parcial de $80 \mathrm{~m}^{2}$ da cobertura da quadra poliesportiva, o CAP1 é composto por telhas de aço galvanizado, calha de chapa metálica galvanizada, condutores verticais $\mathrm{e}$ horizontais de PVC, sistema separador de fluxo inicial ou first flush de $0.3 \mathrm{~mm}$, sifão extravasor, medidor de nível, pontos de coleta e reservatório de 2.460 litros.

O sistema de captação e armazenamento de águas pluviais CAP2 (Figura 2b) localiza-se no bairro Maracanã, no campus da Universidade do Estado do Rio de Janeiro (UERJ), região centro norte da cidade do Rio de Janeiro, totalmente urbanizada, com intenso fluxo de veículos na rua São Francisco Xavier e na Avenida Radial Oeste, que fazem a conexão entre a zona norte e o centro da cidade. Composto por um telhado de fibrocimento com área de captação $30 \mathrm{~m}^{2}$, o CAP2 possui condutores verticais e calhas em PVC, sistema separador de sólidos, first flush primário e secundário, sistema de tratamento preliminar, reservatório com capacidade de armazenamento de 1.000 litros, extravasor, sifão, medidor de nível e pontos de coleta. As amostras de água de águas pluviais são obtidas do first flush primário com capacidade de descartar $0,5 \mathrm{~mm}$ de chuva.

\section{Figura 1 - Área de localização dos sistemas de águas pluviais (CAP1 e CAP2) e da Estação Pluviométrica da Tijuca, na cidade do Rio de Janeiro-RJ}

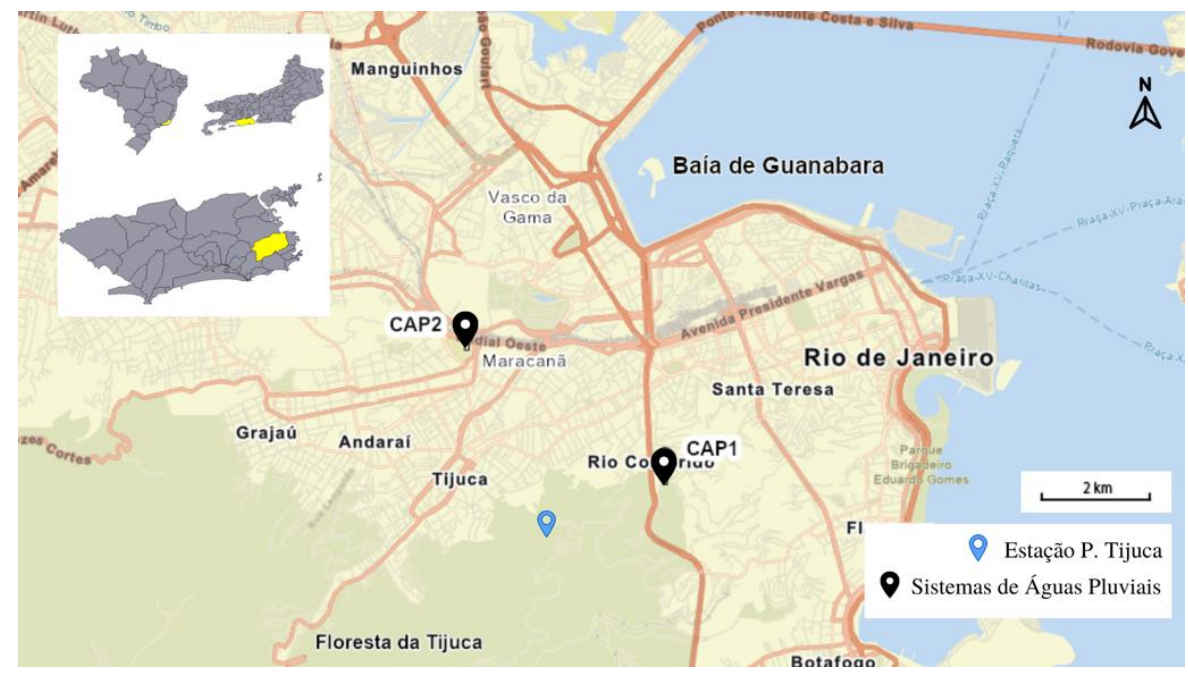

Fonte: autores. 
Tabela 1 - Principais características dos sistemas de águas pluviais na cidade do Rio de Janeiro-RJ

\begin{tabular}{c|c|c|c|c|c|c|c|c}
\hline CAP & Local & Bairro & Região & $\begin{array}{c}\text { População } \\
(*)\end{array}$ & Telhado & Área & $\begin{array}{c}\text { First } \\
\text { flush }\end{array}$ & Reservatório \\
\hline 1 & CAP $\left(^{*}\right)$ & Rio Comprido & Urbana & 43.764 & Metálico & $80 \mathrm{~m}^{2}$ & $0,3 \mathrm{~mm}$ & 2.460 litros \\
\hline 2 & UERJ & Maracanã & Urbana & 25.256 & Fibrocimento & $30 \mathrm{~m}^{2}$ & $0,5 \mathrm{~mm}$ & 1.000 litros \\
\hline
\end{tabular}

(") CAp: Colégio de Aplicação (*) Prefeitura da Cidade do Rio de Janeiro (2010).

Figura 2 - Componentes dos sistemas de qualidade de águas pluviais monitorados

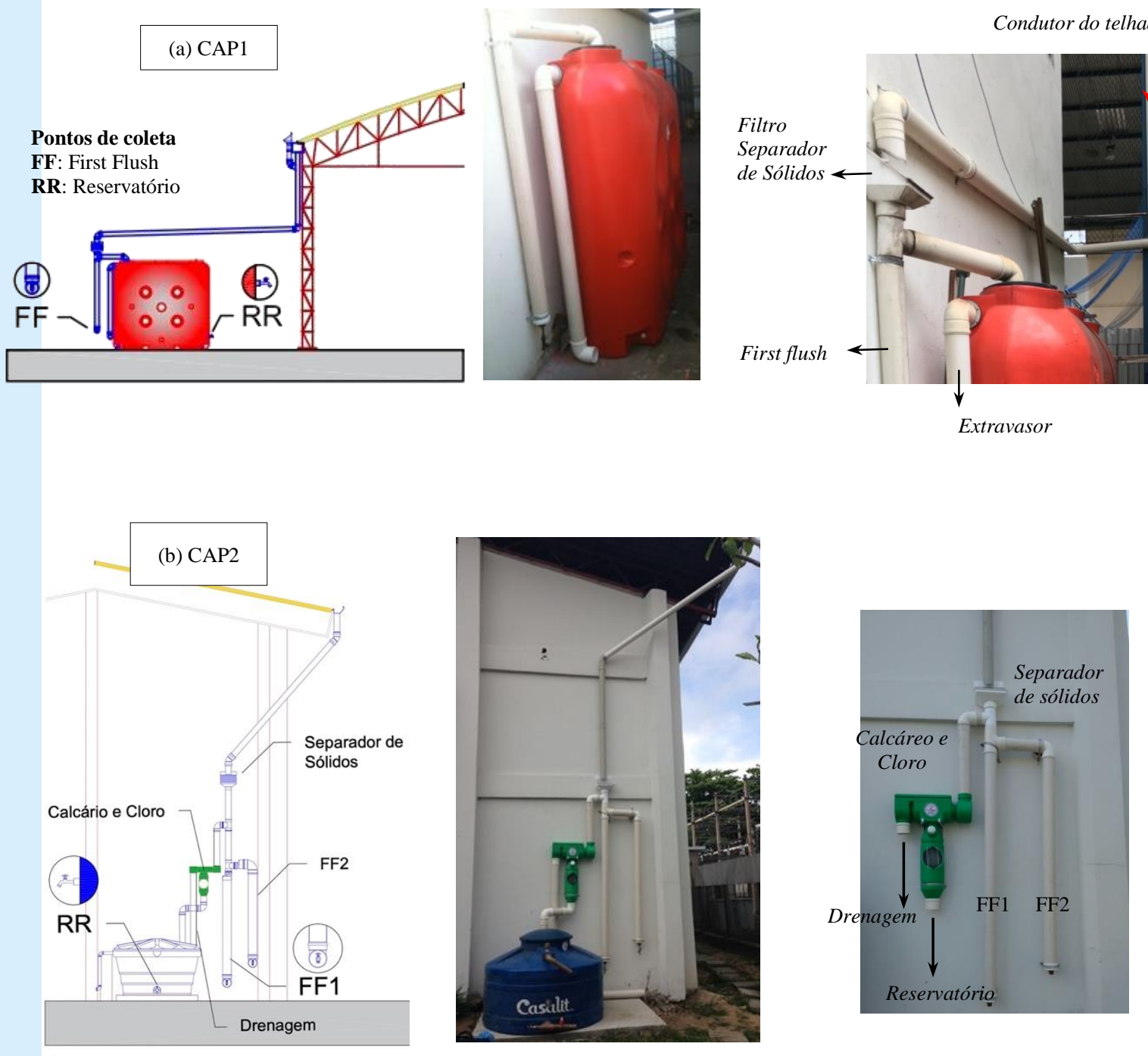

Fonte: autores.

\section{Procedimento amostral}

A caracterização de cada evento de chuva foi feita a partir do primeiro volume armazenado no first flush (FF) e após cada coleta realizada no reservatório, com discretização da precipitação a cada 15 minutos fornecida pelo Sistema Alerta Rio da Prefeitura do Rio de Janeiro. (PREFEITURA
DO RIO DE JANEIRO, 2020) Os dados pluviométricos que constituem este estudo são provenientes de precipitações acima de $1 \mathrm{~mm}$. Foram desconsiderados eventos com intervalos de uma hora de registros sem chuvas. A estação pluviométrica utilizada na obtenção de dados para a realização deste estudo localiza-se na Tijuca, distante $1,60 \mathrm{~km}$ do CAP1 e 2,65 km do CAP2. 
A Figura 3 apresenta as médias da precipitação acumulada em cada mês do histórico entre 1997 e 2019 e dos anos 2017, 2018 e 2019, período analisado neste trabalho. Com um total anual de cerca de $863 \mathrm{~mm}$, o ano de 2017 foi o ano com menor volume precipitado no período analisado, correspondendo a $69 \%$ da média do histórico, de modo que representa o menor volume coletado de amostras. A média mensal em 2017 foi $72 \mathrm{~mm}$, a menor média do período estudado. Em
2018, houve um aumento do volume de chuvas, foram $1.271 \mathrm{~mm}$, e um aumento de $47 \%$ no volume médio de chuva mensal em relação ao ano anterior. $\mathrm{O}$ ano de 2019 também teve um aumento no volume de chuvas anual e mensal, em relação ao ano de 2018, com valores respectivos de 1.804 $\mathrm{mm}$ e $150 \mathrm{~mm}$. No período analisado, a média anual foi $1.313 \mathrm{~mm}$; e a média mensal, $109 \mathrm{~mm}$. (PREFEITURA DO RIO DE JANEIRO, 2020)

Figura 3 - Média mensal histórica de chuva e acumulado mensal do período do estudo

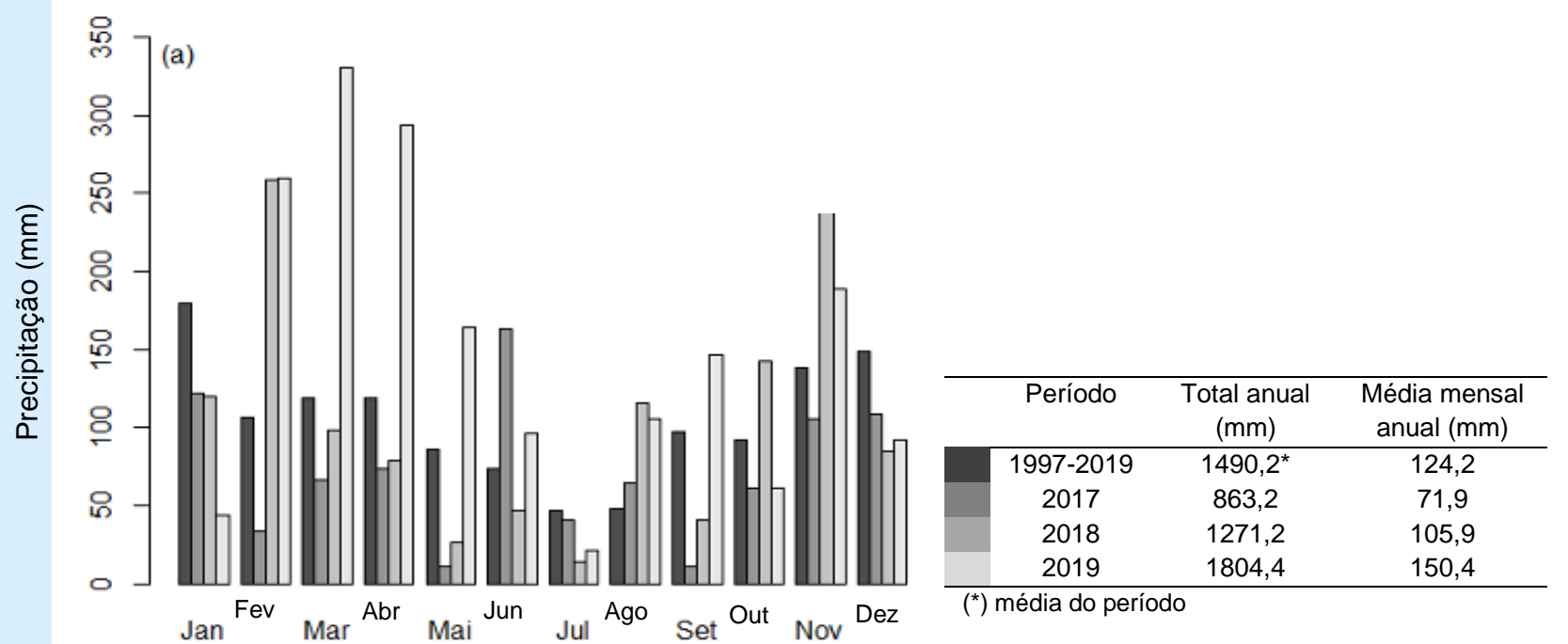

Fonte: Alerta Rio, Prefeitura do Rio de Janeiro, 2020.

Foram coletadas 19 amostras de volumes efetivos precipitados entre janeiro de 2017 e dezembro de 2019 em cada sistema de captação e armazenamento de águas pluviais. A qualidade dessas amostras foi avaliada a partir dos parâmetros: turbidez, sólidos totais dissolvidos (STD), cloreto e alcalinidade, e as análises foram realizadas no Laboratório de Engenharia Sanitária (LES-UERJ), da Faculdade de Engenharia (FEN), da Universidade do Estado do Rio de Janeiro (UERJ). Os parâmetros turbidez, cloreto e alcalinidade foram analisados pelos métodos 2130B, utilizando-se um turbidímetro TECNOPON TB-1000, Método Argentométrico 4500-Cl-B e Método Titulométrico $2320 \mathrm{~B}$, respectivamente, com a referência do Standard Methods for Examination of Water and Wastewater (AMERICAN PUBLIC HEALTH ASSOCIATIONAPHA; AMERICAN WATER WORKS ASSOCIATION-AWWA; WATER ENVIRONMENT FEDERATION-WEF, 2012). A análise dos sólidos totais dissolvidos (STD), em $\mathrm{g} \mathrm{L}^{-1}$, foi desenvolvida por sua relação com a condutividade elétrica (CE), em S/m (McNEIL; COX, 2000), na relação definida pela Equação 1.

$$
S T D=K \times C E
$$

O fator $\mathrm{K}$ pode variar de acordo com o tipo da água, o seu uso e, também, com uma estratificação de valores de condutividade relacionada. A conversão foi realizada de acordo com o coeficiente apresentado pela sonda multiparâmetro Horiba U-50, cuja faixa de condutividade elétrica, abaixo de $0.05 \mathrm{~S} / \mathrm{m}$, tem o valor de $\mathrm{K}$ igual à 0,65. (HORIBA, 2010) Este valor é recomendado para água de irrigação, supondose 0 uso de águas pluviais e de fresh water, consideradas menos nobres. (REINDERS et al., 2010; RUSYDI, 2018)

A NBR no 15527/2019 dispõe de valores restritivos de determinados parâmetros de 
qualidade de água, como a turbidez. Para avaliação da qualidade das amostras em relação aos parâmetros sólidos totais dissolvidos (STD) e cloreto, foi adotada como referência a Portaria de Consolidação no 888/2021. (BRASIL, 2021)

O método proposto para integração dos aspectos qualitativos e quantitativos baseia-se nos resultados de análises de determinados parâmetros da água da chuva e faz a associação destes com o volume de escoamento superficial armazenado nos sistemas de águas pluviais.

A mensuração da poluição difusa é difícil, pois esta ocorre de forma aleatória e incorporada ao ciclo da água, além de depender de fatores físicos relacionados à área de estudo, tais como elementos meteorológicos e o tipo de uso e ocupação do solo. Desse modo, esse tipo de poluição consiste em poluentes atmosféricos depositados e acumulados nas superfícies impermeáveis que são arrastados pela água. (FREITAS, 2013 apud RIGHETTO; GOMES; FREITAS, 2017)

Para análise da variação da taxa de massa de poluentes que é transportada ao longo do escoamento superficial pelo telhado das edificações, foram utilizadas as curvas $M(V)$, a partir do gráfico da fração cumulativa de massa total de poluentes versus a fração cumulativa do volume total escoado. $O$ gráfico das curvas $M(V)$ permite também analisar de forma adimensional eventos de diferentes localidades e características, sendo a representação matemática obtida a partir da Equação (2), com N medições da vazão $Q_{i}$ e da concentração de poluente $\mathrm{C}_{\mathrm{i}}$ em cada intervalo de tempo $\Delta \mathrm{t}_{\mathrm{i}}$. Devese considerar que tanto a vazão quanto a concentração variam linearmente entre duas medições (BERTRAND-KRAJEWSKI; CHEBBO; SAGET, 1998).

$$
\frac{\sum_{i=1}^{j} C_{i} Q_{i} \Delta t_{i}}{\sum_{i=1}^{N} C_{i} Q_{i} \Delta t_{i}}=f\left(\frac{\sum_{i=1}^{j} Q_{i} \Delta t_{i}}{\sum_{i=1}^{N} Q_{i} \Delta t_{i}}\right)=f\left(\frac{\sum_{i=1}^{j} V_{i}}{\sum_{i=1}^{j} V_{i}}\right)
$$

Sendo $N$, o número total de medições,

$J$, o índice de 1 a $N$, e

$V_{i}$, o volume escoado durante o intervalo de tempo $\Delta t_{\text {i. }}$

De forma global, o impacto de cargas difusas no escoamento pluvial é avaliado a partir do cálculo da sua concentração média no evento (CME), cujo valor representa a relação entre a massa de poluentes transportada e o volume de escoamento superficial (Equação 3).

$$
C M E=\frac{M}{V}=\frac{\int_{0}^{t} C_{t} Q_{t} d t}{\int_{0}^{t} Q_{t} d t}=\frac{\sum C_{t} Q_{t} \Delta t}{\sum Q_{t} \Delta t}
$$

As curvas $M(V)$ representam as frações de massa de poluente removidas relativas a cada fração de volume escoado ao longo de um período. O eixo da abscissa é a fração de volume escoado e o eixo da ordenada é a fração de massa de poluente. Quando a curva do parâmetro estiver acima do limite do bissetor, pode-se considerar a ocorrência do fenômeno first flush. Assim, a magnitude desse fenômeno pode ser avaliada pelo seu afastamento em relação à linha divisória dada pelo bissetor. (FERREIRA, 2008)

\section{RESULTADOS E DISCUSSÃO}

Realizaram-se análises do comportamento das curvas $\mathrm{M}(\mathrm{V})$ dos sistemas CAP1 e CAP2 em relação aos parâmetros turbidez, sólidos totais dissolvidos (STD), cloreto e alcalinidade nos pontos first flush (FF) (Figura 4) e reservatório (RR) (Figura 5). Os parâmetros cloreto e alcalinidade em quase todos os pontos mantiveram-se acima do bissetor e com uma maior concentração de poluentes nos primeiros volumes escoados (Figuras $4 b$ e $5 b$ ).

O sistema de captação e armazenamento de águas pluviais CAP2 mostrou uma maior eficiência na remoção de carga de lavagem do parâmetro turbidez nos volumes iniciais precipitados do que 0 obtido no sistema CAP1, pelo fato de a curva se manter acima da do bissetor e pela maior proporção de massa acumulada em relação ao volume efetivo precipitado (Figura 4a). A carga de sólidos totais dissolvidos (STD) do sistema CAP1 mostra um comportamento análogo ao do CAP2, não sendo evidenciada a remoção na lavagem de poluentes nos primeiros minutos de precipitação em nenhum dos sistemas, porque as curvas estão próximas ou abaixo do bissetor. Assim como os STD, o cloreto e a alcalinidade apresentaram resultados de contração do poluente pelo volume escoado correlativo nos dois sistemas (Figura 4b). 
Figura 4 - Curvas M(V) do ponto first flush no CAP1 e CAP2 dos parâmetros (a) turbidez e STD e (b) cloreto e alcalinidade
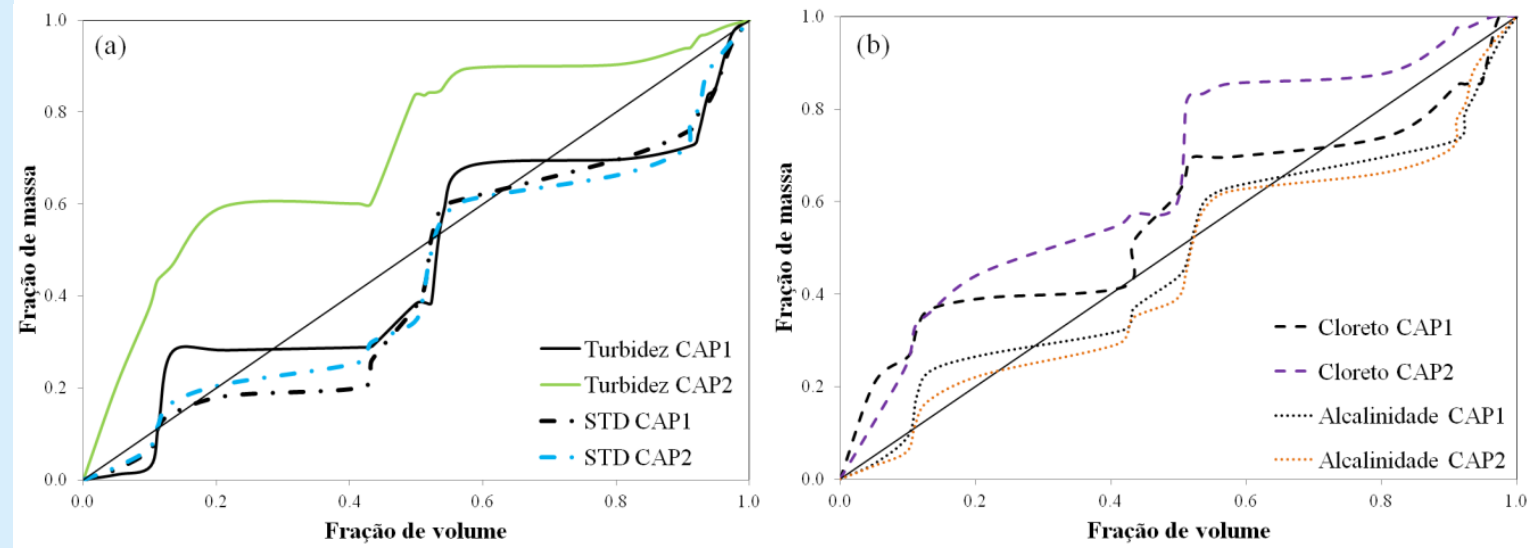

Fonte: autores.

Figura 5 - Curvas $M(V)$ do ponto reservatório no CAP1 e CAP2 dos parâmetros (a) turbidez e STD e (b) cloreto e alcalinidade

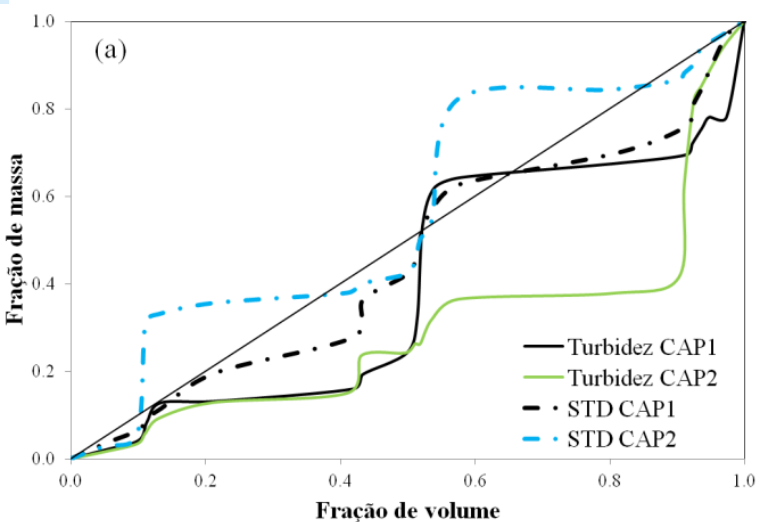

Fonte: autores.

Em geral, o resultado encontrado para a carga de poluentes do efluente do reservatório (Figura 5) apresenta comportamento similar aos obtidos para a remoção observada nas curvas $\mathrm{M}(\mathrm{V})$ do ponto do first flush, com uma pequena variação mostrando melhor eficiência na lavagem dos volumes iniciais precipitados nas curvas dos STD e do cloreto no sistema CAP2, com exceção da turbidez, que expõe uma alta concentração de poluentes na fração final do volume.

O resultado relativo à concentração média do evento (CME) foi obtido considerando-se cada um dos três anos em foco separadamente, sendo uma medida de avaliação da carga de poluição difusa depositada na superfície dos telhados e afluentes aos sistemas de captação e armazenamento de água de chuva dos CAP1 e CAP2 (Figura 6).

A Figura 6(a) indica a concentração média do parâmetro turbidez do ponto first flush, a qual mostra um valor bastante superior em relação ao ponto de coleta no reservatório. A diferença entres

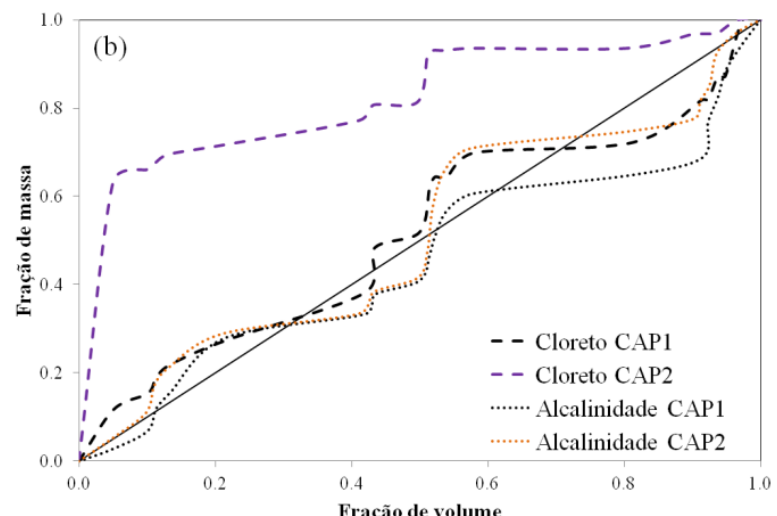

os valores desse parâmetro evidencia a importância do bloqueio inicial dos volumes efetivos precipitados como uma medida de redução da concentração e da carga de poluentes depositados na superfície do telhado.

Os STD (Figura 6b) apresentam resultados pouco representativos, em relação à sua concentração média em todos os três anos analisados. No ponto RR CAP2, a concentração média de cloreto apresentou uma elevação em 2017 (Figura 6c), explicado pela adição de pastilhas de cloro no sistema de armazenamento. Os resultados elevados da concentração média de alcalinidade (Figura 6d) no CAP2 são justificados provavelmente em decorrência de características próprias do fibrocimento, material do telhado.

A qualidade das águas pluviais armazenadas em um sistema pode ser influenciada pelas vazões afluentes ao reservatório. Dependendo da vazão, a diluição dos poluentes pode ser favorecida ou ficar prejudicada. Dessa forma, exceto em relação 
à turbidez no ponto FF CAP2 ao cloreto no ponto RR CAP2, os anos 2017 e 2019 apresentaram resultados semelhantes no que se refere à concentração dos parâmetros estudados, apesar de haver uma diferenciação nos volumes precipitados nesses anos.

A tabela 2 expõe a estatística descritiva da série histórica dos parâmetros de qualidade de água de chuva estudados dos dois sistemas, CAP1 e CAP2.

Figura 6 - Representação da concentração média anual de (a) turbidez, (b) STD, (c) cloreto e (d) alcalinidade nos pontos first flush (FF) e reservatório (RR)
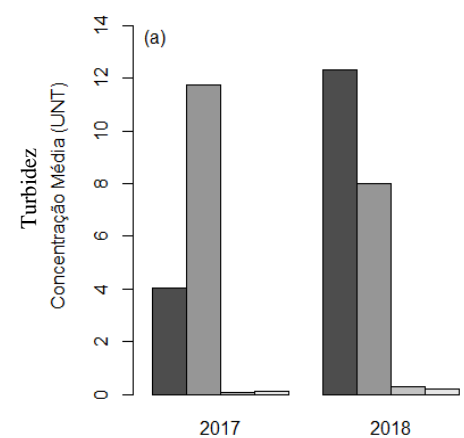

Anos

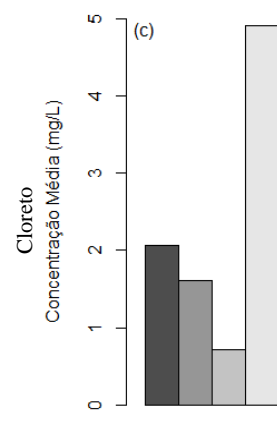

2017

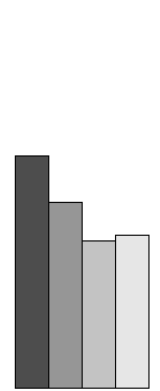

2018

Anos
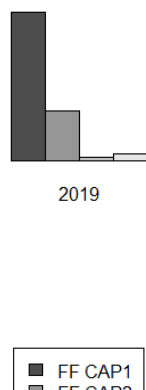
FF CAP2 $\square$ RR CAP1
$\square$ RR CAP2

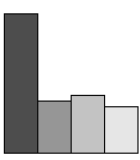

2019

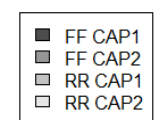

Fonte: autores.

De acordo com os desvios-padrão obtidos, pode-se observar que, no geral, as análises de amostras de volumes do first flush (FF) apresentaram maior volatilidade. Desse modo, é possível afirmar que, em um evento chuvoso, o descarte inicial é responsável por carrear a maior parte dos poluentes atmosféricos e dos depositados na superfície de captação. Constatase assim o fenômeno do first flush. (HVITVEDJACOBSEN; VOLLERTSEN; NIELSEN, 2010; FERREIRA, 2008)

Os poucos dias secos antecedentes representaram baixo potencial de deposição úmida no telhado, o que favoreceu o aparecimento de valores reduzidos de determinados parâmetros, como turbidez e sólidos totais dissolvidos (STD).

Dos parâmetros analisados, a turbidez apresentou resultados acima do permitido pela NBR no 15527/2019 (ABNT, 2019) e pela Portaria
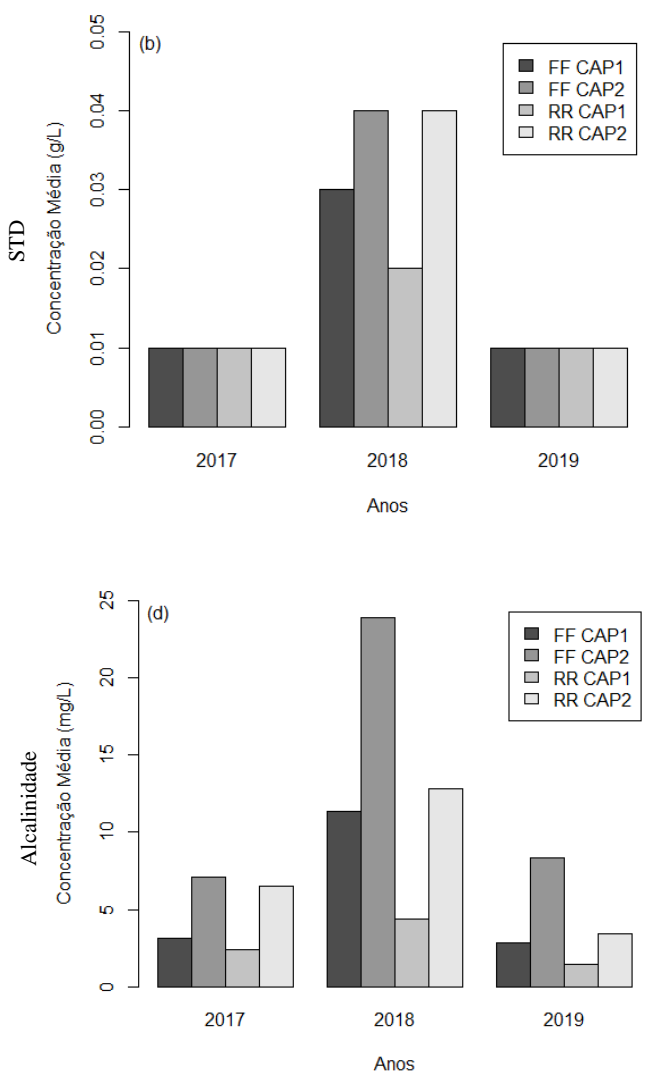

de Consolidação № 888/2021 (BRASIL, 2021), como destacado em negrito na Tabela 2. Foram observados valores médio e máximo de turbidez acima do permitido no ponto de coleta do first flush (FF). No ponto do reservatório (RR), houve redução desse parâmetro até o limite máximo permitido. Demais parâmetros analisados atenderam às restrições da norma e da Portaria de Consolidação no 888/2021 - Anexo XX (BRASIL, 2021).

A consolidação dos resultados das análises dos parâmetros turbidez, sólidos totais dissolvidos (STD), cloreto e alcalinidade, obtidos entre janeiro de 2017 e dezembro de 2019, a partir de uma avaliação da distribuição empírica dos dados coletados nos pontos do first flush (FF) e reservatório (RR), nos sistemas CAP1 e CAP2 estão apresentados na Figura 7. 
Tabela 2 - Estatística descritiva de parâmetros de qualidade de água de chuva

\begin{tabular}{|c|c|c|c|c|c|c|}
\hline \multirow{2}{*}{$\begin{array}{c}\text { Parâmetro } \\
\text { (unidade) }\end{array}$} & \multicolumn{4}{|c|}{ CAP1 (CAP2) } & \multirow{2}{*}{$\begin{array}{c}\text { NBR } \mathrm{n}^{\circ} \\
15527 / 20 \\
19 \\
\end{array}$} & \multirow{2}{*}{$\begin{array}{c}\text { Port. Cons } \\
n^{\circ} \\
888 / 2021 \\
\end{array}$} \\
\hline & Máx. & Mín. & Méd. & D.P. & & \\
\hline $\begin{array}{l}\text { Precipitação } \\
(\mathrm{mm})\end{array}$ & $40,4(40,4)$ & $1,6(1,6)$ & $10,4(11,6)$ & $11,2(12,3)$ & --- & --- \\
\hline $\begin{array}{c}\text { Dias secos } \\
\text { antecedentes } \\
\text { (dias) }\end{array}$ & $22,0(21,0)$ & $0(0)$ & $6,0(5,0)$ & $5,4(5,4)$ & --- & --- \\
\hline $\begin{array}{c}\text { Turbidez FF } \\
\text { (UNT) }\end{array}$ & $129,0(91,0)$ & $0,00(0,08)$ & $34,97(20,43)$ & $39,15(28,84)$ & \multirow{2}{*}{$<5$} & \multirow{2}{*}{$<5$} \\
\hline $\begin{array}{c}\text { Turbidez RR } \\
\text { (UNT) }\end{array}$ & $2,7(3,70)$ & $0,0(0,0)$ & $0,65(0,92)$ & $0,75(1,02)$ & & \\
\hline STD FF $\left(g^{-1}\right)$ & $0,09(0,11)$ & $0,014(0,034)$ & $0,02(0,064)$ & $0,75(0,02)$ & --- & \multirow{2}{*}{$<0,5$} \\
\hline STD RR $\left(\mathrm{g} \mathrm{L}^{-1}\right)$ & $0,06(0,42)$ & $0,011(0,023)$ & $0,027(0,079)$ & $0,01(0,11)$ & --- & \\
\hline $\begin{array}{c}\text { Cloreto FF (mg } \\
\left.\mathrm{L}^{-1}\right)\end{array}$ & $25,2(16,87)$ & $0,0(0,0)$ & $6,34(4,05)$ & $6,96(4,49)$ & --- & \multirow{2}{*}{$<250$} \\
\hline $\begin{array}{c}\text { Cloreto RR (mg } \\
\left.\mathrm{L}^{-1}\right)\end{array}$ & $6,8(88,2)$ & $0,0(0,0)$ & $2,82(7,34)$ & $2,32(19,91)$ & --- & \\
\hline $\begin{array}{c}\text { Alcalinidade FF } \\
\left(\mathrm{mg} \mathrm{L}^{-1}\right)\end{array}$ & $27,27(63,99)$ & $9,18(22,32)$ & $17,02(41,11)$ & $5,11(10,35)$ & --- & --- \\
\hline $\begin{array}{c}\text { Alcalinidade RR } \\
\left(\mathrm{mg} \mathrm{L}^{-1}\right)\end{array}$ & $19,98(49,68)$ & $4,59(13,80)$ & $8,48(26,18)$ & $3,45(9,66)$ & --- & --- \\
\hline
\end{tabular}

Legenda: Máx.: máximo; Mín.: mínimo, Méd.: média; D.P.: desvio-padrão; FF: first flush; RR: reservatório.

Nota: os valores entre parênteses referem-se aos resultados obtidos no CAP2. Em negrito, valores não atendidos, conforme as referências utilizadas.

Figura 7 - Qualidade da água da chuva obtida a partir da série de dados coletados de 2017 a 2019 para (a) turbidez, (b) STD, (c) cloreto e (d) alcalinidade nos pontos first flush (FF) e reservatório (RR)
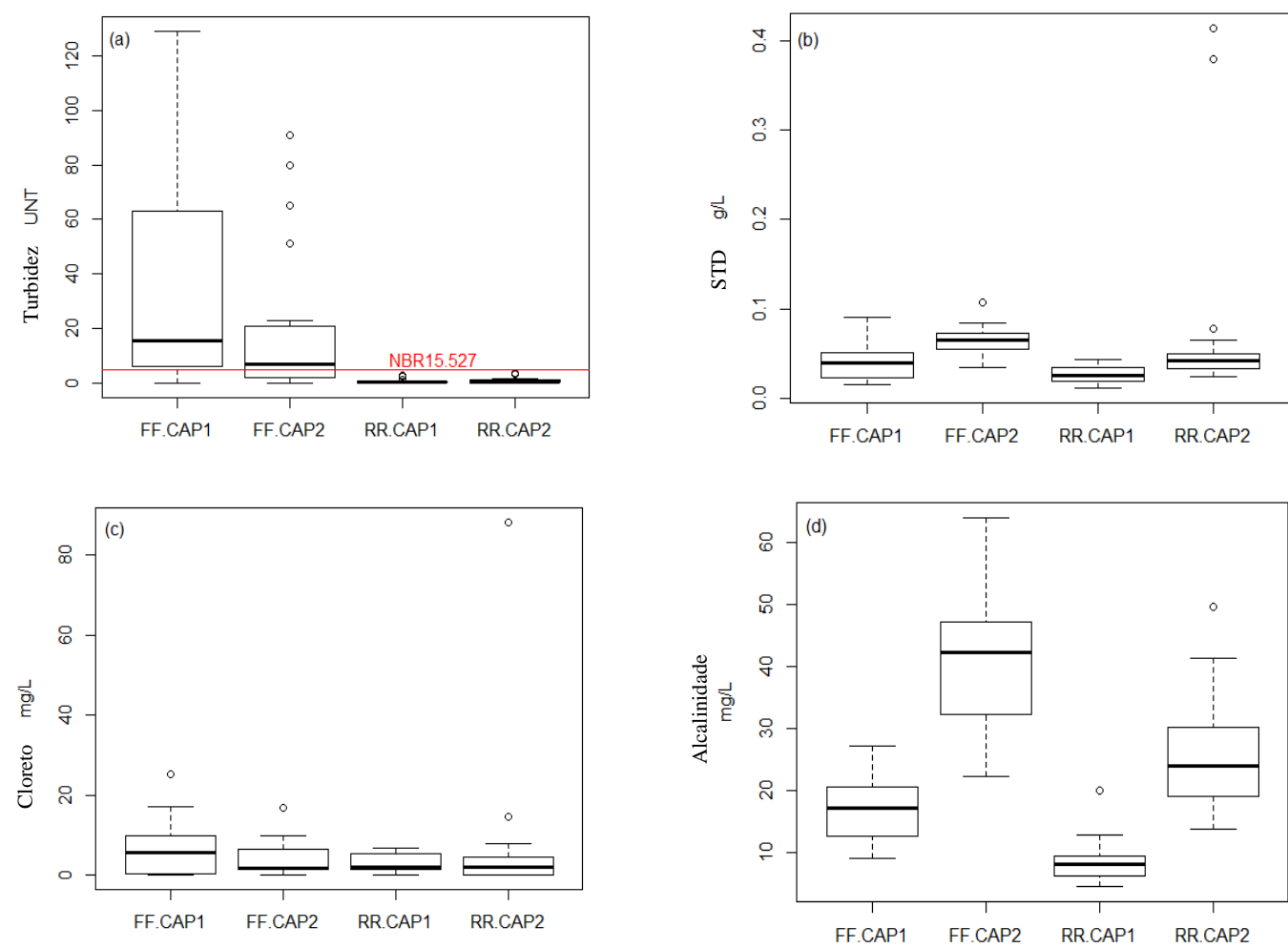

Fonte: autores. 
Os resultados obtidos para turbidez no ponto first flush (FF), em ambos os sistemas CAP1 e CAP2, foram significativos, estando acima do estabelecido pela NBR no 15527/2019 (Figura 8a), com valores mais elevados no CAP1. No ponto do reservatório (RR) ocorreu similaridade na distribuição dos dados nos dois locais. Pode-se associar a diminuição deste parâmetro do ponto montante (FF) para o ponto jusante (RR) à eficiência da primeira lavagem da chuva que ocorre no sistema de armazenamento. Isso justifica a melhoria na qualidade da água de chuva e torna maior o seu aproveitamento. (GIKAS; TSIHRINTZIS, 2012)

Os sólidos totais dissolvidos (STD) apresentaram resultados abaixo de $0,5 \mathrm{~g} \mathrm{~L}^{-1}$, considerado o valor máximo exigido pela Portaria de Consolidação no. 888/2021 - Anexo XX (Figura 8b). De modo geral, os sistemas apresentaram comportamento semelhante, com pequena elevação no sistema do CAP2. As análises de cloreto ficaram abaixo de $250 \mathrm{mg} / \mathrm{L}$, valor permitido pela Portaria de Consolidação ํo 888/2021 Anexo XX (Figura 8c).

O sistema do CAP1 apresentou maiores valores de concentração em comparação ao CAP2, com ligeira melhora na qualidade da água armazenada no ponto do reservatório. Em ambos os sistemas CAP1 e CAP2, a alcalinidade diminuiu na medida em que percorreu o sistema de montante para jusante (Figura 8d), ou do ponto do first flush (FF) para o ponto de armazenamento no reservatório (RR). Não há valores de referência para alcalinidade na NBR o 15527/2019 (ABNT, 2019) e na Portaria de Consolidação no $888 / 2021$ - Anexo XX. (BRASIL, 2021)

O impacto do material da cobertura do telhado de captação de água de chuva na qualidade da água armazenada no reservatório pôde ser avaliado por meio dos parâmetros turbidez, cloreto e alcalinidade. Os resultados dessa análise constam na Tabela 3.

\section{Tabela 3 - Resultados de parâmetros de qualidade de água de chuva armazenada no reservatório,} em função do material do telhado

\begin{tabular}{|c|c|c|c|c|c|c|c|c|c|c|c|c|}
\hline Referência & \multicolumn{3}{|c|}{ CAP1 } & \multicolumn{3}{|c|}{ Tordo (2004) } & \multicolumn{3}{|c|}{ CAP2 } & \multicolumn{3}{|c|}{ Tordo (2004) } \\
\hline Tipo telhado & \multicolumn{3}{|c|}{ metálico } & \multicolumn{3}{|c|}{ metálico } & \multicolumn{3}{|c|}{ fibrocimento } & \multicolumn{3}{|c|}{ fibrocimento } \\
\hline $\begin{array}{l}\text { Parâmetro/ } \\
\text { Estatística }\end{array}$ & Máx & Min & Méd & Máx & Min & Méd & Máx & Min & Méd & Máx & Min & Méd \\
\hline Turbidez (UNT) & 2,70 & 0,00 & 0,65 & 11,36 & 0,24 & 2,13 & 3,70 & 0,00 & 0,92 & 1,78 & 0,28 & 2,34 \\
\hline Cloreto (mg/L) & 6,80 & 0,00 & 2,82 & 15,51 & 2,82 & 6,85 & 88,20 & 0,00 & 7,34 & 11,28 & 1,41 & 5,09 \\
\hline Alcalinidade (mg/L) & 20,00 & 4,59 & 8,48 & 12,00 & 8,00 & 9,71 & 49,68 & 13,80 & 26,18 & 55,96 & 18,00 & 37,06 \\
\hline
\end{tabular}

Legenda: Máx: máximo; Mín: mínimo, Méd: média.

As telhas de fibrocimento são capazes de neutralizar a acidez de volumes efetivamente precipitados de modo mais eficiente do que telhas metálicas. Segundo Tordo (2004), os resultados da alcalinidade das águas de chuva captadas de telhados de fibrocimento têm mostrado valores mais elevados se comparados com os das telhas metálicas. No caso do aço galvanizado ou das telhas metálicas, a melhor qualidade da água de chuva coletada no reservatório provavelmente se deve também à influência da luz ultravioleta e à alta temperatura. (LEE; BAK; HAN, 2012)

No período das análises realizadas neste estudo, de 2017 a 2019, os valores médios de alcalinidade foram significativamente maiores nas águas provenientes de telhado de fibrocimento, se comparados aos obtidos com amostras de água coletada de telhas metálicas. De modo contrário, a turbidez e o cloreto apresentaram valores mais elevados com os telhados metálicos.

\section{CONCLUSÃO}

Este trabalho avaliou a eficiência na remoção de poluentes atmosféricos de dois sistemas de captação e armazenamento de águas pluviais, denominados CAP1 e CAP2, e concluiu que:

(i) as características climáticas na região do estudo, como o tipo da precipitação, assim como as características físicas do sistema, como material de captação da cobertura da edificação, influenciam na variabilidade da remoção de poluentes depositados na superfície do telhado; 
(ii) a qualidade da água armazenada no sistema de coleta de água de chuva melhora significativamente de montante para jusante, na medida em que são instalados sistemas de retenção de sedimentos e descarte dos volumes iniciais precipitados, sobretudo na correção da carga de sólidos totais dissolvidos e na neutralização química da chuva;

(iii) as águas armazenadas pelos dois sistemas possuem um potencial de uso para fins não potáveis, tendo em vista que, se houver 0 descarte dos volumes iniciais precipitados, os resultados de turbidez, de sólidos totais dissolvidos e cloreto no ponto de aproveitamento do reservatório mostram conformidade com a NBR o 15527/2019 (ABNT, 2019) e à Portaria de Consolidação no 888/2021 - Anexo XX (BRASIL, 2021);

(iv) as curvas $M(V)$ e a concentração média do evento (CME) indicaram o potencial de lavagem dos poluentes iniciais precipitados $\mathrm{e}$ da carga de poluição nos sistemas de drenagem urbana.

\section{AGRADECIMENTOS}

Ao Conselho Nacional de Pesquisa e Desenvolvimento Científico (CNPq) e à Fundação de Amparo à Pesquisa do Estado do Rio de Janeiro (FAPERJ), pela concessão de bolsa de Iniciação Científica e Tecnológica, e à Universidade do Estado do Rio de Janeiro, pelo apoio na elaboração deste trabalho.

\section{REFERÊNCIAS}

AMERICAN PUBLIC HEALTH ASSOCIATION; AMERICAN WATER WORKS ASSOCIATION; WATER ENVIRONMENT FEDERATION. Standard Methods for the Examination of Water and Wastewater. 22 ed. Washington: APHA, 2012.

ASSOCIAÇÃO BRASILEIRA DE NORMAS TÉCNICAS. NBR 15527: Aproveitamento de água de chuva de coberturas em áreas urbanas para fins não potáveis Requisitos. Rio de Janeiro, 2019.

BERTRAND-KRAJEWSKI, J.; CHEBBO, G.; SAGET, A. Distribution of pollutant mass vs volume in stormwater discharges and first flush phenomenon. Water

Resource, [S. I.], v. 32, n. 8, p. 2341-2356, 1998.

BRASIL. Ministério da Saúde. Portaria de Consolidação GM/MS n. 888/2021 - Anexo XX, de 4 de maio de
2021. Altera o Anexo XX da Portaria de Consolidação GM/MS n 5 , de 28 de setembro de 2017, para dispor sobre os procedimentos de controle e de vigilância da qualidade da água para consumo humano e seu padrão de potabilidade. Diário Oficial da União, Brasília, DF, Poder Executivo, 07 mai. 2021. seção 1, p. 127.

DIDONET, T. T. S. C. Relações entre precipitação, escoamento e sedimentos suspensos na bacia do Rio das Antas, Goiás. 2017. 101 f. Dissertação (Mestrado em Engenharia Ambiental e Sanitária) Universidade Federal de Goiás, Goiânia, 2017.

FERREIRA, L. C. A. Variação da qualidade da água do escoamento superficial de duas bacias de drenagem de Natal/RN - Brasil. 100 f. Dissertação (Mestrado em Engenharia Sanitária) - Universidade Federal do Rio Grande do Norte, Natal, 2008.

GIKAS, G. D., TSIHRINTZIS, V. A. Assessment of water quality of first flush roof runoff and harvested rainwater. Journal of Hydrology, [S. I.], n. 466-467, p.115-126, October 2012. Disponível em: <https://doi.org/10.1016/j.jhydrol.2012.08.020>. Acesso em: 27 jul. 2021.

GIMENES, J. C. Captação e aproveitamento de água de chuva: estudo experimental da qualidade de água de um telhado verde e de um telhado convencional. Trabalho de Conclusão de Curso. $102 \mathrm{f}$. (Graduação em Engenharia Civil) - Escola Politécnica, Universidade Federal do Rio de Janeiro, Rio de Janeiro, 2017.

GOMES, K. M. F. Modelagem da poluição difusa em uma bacia de drenagem urbana. 2014. $76 \mathrm{f}$.

Dissertação (Mestrado em Engenharia Ambiental) Universidade Federal do Rio Grande do Norte, Natal, 2014. Disponível em:

<https://repositorio.ufrn.br/jspui/bitstream/123456789/1 9602/1/KalineMurieIDeFigueiredoGomes_DISSERT.pdf >. Acesso em: 29 mar. 2021.

GUNAWARDANA, C. et al. Role of Solids in Heavy Metals Buildup on Urban Road Surfaces. Journal of Environmental Engineering, [S.I.] n. 138(4), p. 490498, 2012. Disponível em:

<https://doi.org/10.1061/(asce)ee.1943-7870.0000487>. Acesso em: 27 julho de 2021.

HAUPT, J. P. O. Metodologia para avaliação do potencial de produção de poluição difusa: estudo de caso da bacia do Rio Jundiaí. 2009. Dissertação (Mestrado em Engenharia Hidráulica) - Escola Politécnica, Universidade de São Paulo, São Paulo, 2009. Disponível em: $<$ https://doi.org/10.11606/D.3.2009.tde-20072009173457>. Acesso em: 27 jul. 2021. 
HORIBA LTD. Multi Water Quality Checker U-50

Series: Instruction Manual. Japan, 2010.

HVITVED-JACOBSEN, T.; VOLLERTSEN, J.; NIELSEN, A. H. Urban and Highway Stormwater Pollution: Concepts and Engineering. London: New York: CRC Press, 2010.

LEE, J. Y.; BAK, G.; HAN, M. Quality of roof-harvested rainwater - Comparison of different roofing materials, Environmental Pollution, [S. I.], v. 162, p. 422-429.

2012. Disponível em:

<https://doi.org/10.1016/j.envpol.2011.12.005>. Acesso em: 27 de jul. 2021.

McNEIL, V. H.; COX, M. E. Relationship between conductivity and analysed composition in a large set of natural surface-water samples, Queensland, Australia. Environmental Geology, [S. I.], n. 39(12), p. 13251333, 2000. Disponível em: <https://doi.org/10.1007/s002549900033>. Acesso em: 27 de jul.de 2021.

NAKADA, L. Y., MORUZZI, R. B. Variabilidade qualitativa de águas pluviais coletadas em telhado e sua importância na concepção do sistema de tratamento. Engenharia Ambiental e Sanitária, Rio de Janeiro, v.19, n.1, p.1-9, 2014. Disponível em: <https://doi.org/10.1590/S1413-41522014000100001>. Acesso em: 27 jul. 2021.

OUYANG, W. et al. The washing effect of precipitation on particulate matter and the pollution dynamics of rainwater in downtown Beijing. Science of the Total Environment, [S. I.], n. 505, p. 306-314, 2015. Disponível em: <https://doi.org/10.1016/j.scitotenv.2014.09.062>. Acesso em: 27 jul. 2021.

PINTO, C. C. Importância da avaliação de dados de concentração e de carga de poluentes em bacias hidrográficas: exemplo da Bacia do Rio Verde. 2016. 189 f. Dissertação (Mestrado em Saneamento, Meio Ambiente e Recursos Hídricos) - Universidade Federal de Minas Gerais, Belo Horizonte, 2016.

PREFEITURA DA CIDADE DO RIO DE JANEIRO. Fluxo Veicular Médio em Dias Úteis. Rio de Janeiro, 2017.

PREFEITURA DA CIDADE DO RIO DE JANEIRO. Data.Rio: bairros cariocas. Rio de Janeiro, 2018. Disponível em: <pcrj.maps.arcgis.com/apps/MapJournal/index.html>. Acesso em: 14 jan. 2021.
PREFEITURA DO RIO DE JANEIRO. Alerta Rio.

Sistema de Alerta Rio da Prefeitura do Rio de Janeiro.

Sistema de Alerta de Chuvas Intensas e de Deslizamentos em Encostas da Cidade do Rio de Janeiro. Rio de Janeiro, 2020. Disponível em: <http://alertario.rio.rj.gov.br/>. Acesso em: 14 jan. 2021.

REINDERS F. B. et al. Standards and Guidelines for Improved Efficiency of Irrigation Water Use from Dam Wall Release to Root Zone Application: Supplementary Information. Report n. TT 467/10. Pretoria, South Africa: Water Research Commission, 2010.

RIGHETTO, A. M.; GOMES, K. M.; FREITAS, F. R. S. Poluição difusa nas águas pluviais de uma bacia de drenagem urbana. Engenharia Sanitária e Ambiental, Rio de Janeiro, v. 22, n. 6, p. 1109-1120, dez. 2017. Disponível em: <http://dx.doi.org/10.1590/s141341522017162357>. Acesso em: 27 jul. 2021.

RUSYDI, A. F. Correlation between conductivity and total dissolved solid in various type of water: a review. IOP Conference Series: Earth and Environmental Science, [S.I.], v. 118 012019, 2018. Disponível em: <https://iopscience.iop.org/article/10.1088/17551315/118/1/012019>. Acesso em: 27 jul. 2021.

SILVA, N. N. da; CRUZ, J. A. da; AMARAL, L. G. H do. Dimensionamento de reservatórios de lote para redução do volume de escoamento superficial na cidade de Barreiras-BA. In: SIMPÓSIO DE RECURSOS HÍDRICOS DO NORDESTE. 13., 2016, Aracaju, SE. Anais [...]. Porto Alegre: Associação Brasileira de Recursos Hídricos, 2016. Disponível em: <http://abrh.s3.amazonaws.com/Eventos/Trabalhos/27/ PAP021849.pdf>. Acesso em: 27 jul. 2021.

TORDO, O. C. Caracterização e avaliação do uso de águas de chuva para fins potáveis. 2004. Dissertação (Mestrado em Engenharia Ambiental) Universidade Regional de Blumenau, Blumenau, 2004.

VASCONCELOS, A. F.; MIGUEZ, M. G.; VAZQUEZ, E. G. Critérios de projeto e benefícios esperados da implantação de técnicas compensatórias em drenagem urbana para controle de escoamentos na fonte, com base em modelagem computacional aplicada a um estudo de caso na zona oeste do Rio de Janeiro.

Engenharia Sanitária e Ambiental, Rio de Janeiro, n. 21(4), p. 655-662, 2016. Disponível em: <https://doi.org/10.1590/s1413-41522016146469>. Acesso em: 27 julho de 2021. 